\title{
Influence of the thickness absorbing film on the PETN ignition threshold by a laser pulse
}

\author{
Vadim A. Dolgachev ${ }^{1}$, Alexander V. Khaneft ${ }^{1,2 a}$ \\ ${ }^{1}$ Kemerovo State University, 650043 Kemerovo, Russia \\ ${ }^{2}$ National Research Tomsk Polytechnic University, 634050 Tomsk, Russia
}

\begin{abstract}
Numerical simulation of the PETN ignition by a film, which is heated by a laser pulse was conducted. There are shown that dependence of threshold energy of ignition of PETN by a laser pulse has a linear dependence from the thicknesses of the absorbing film. Calculations shown that critical the temperature on the boundary of two materials by the end of a laser pulse with threshold density doesn't depend from the thickness of the absorbing film. The ignition delay time of PETN by the thick film less than the ignition delay time of PETN by the thin film. The reason is that the thicker contain more heat then in the thinner one.
\end{abstract}

\section{Introduction}

In recent years significantly increased the number of works on the experimental study and numerical simulation of ignition of condensed explosives by a laser radiation, as well as by heat sources with a limited power capacity [1-15]. This is caused by the prospects of practical use of laser initiation of energetic materials, and clarification of the mechanism of ignition of explosives.

Simulation of ignition of energetic materials, which absorb the laser radiation by inclusions or films, usually don't take into account the distribution of the light flux on an inclusion or a film. It is true in case of metal particles in the volume or metal film deposited of the surface of explosives. It is assumed that the temperature distribution in the inclusion or in the deposited film is uniform due to the high thermal conductivity metal. In the case of inclusions or films deposited on the surface of explosives, for example, metal oxides, it is necessary to take into account the distribution of light energy along the thickness of the absorbing film in the range of wavelengths $\lambda=1060 \div 1070 \mathrm{~nm}$ when exposed, for example, to a neodymium laser or a fiber laser YLS-150. This is due to the fact that the thermal conductivity of oxides is significantly smaller than that of a metal.

The aim of this work is to determine the dependence of the minimum ignition energy of PETN by a laser pulse, depending on the thickness of the absorbing film of copper oxide.

${ }^{a}$ Corresponding author: khaneft@kemsu.ru 


\section{Problem statement}

Consider a two-layer heterosystem: a film absorbing laser radiation and a condensed explosive heated by the film (Figure 1). One-dimensional equations of thermal conductivity for the two-layer system can be written as:

$$
\begin{gathered}
\rho_{1} c_{1} \frac{\partial T_{1}}{\partial t}=\lambda_{1} \frac{\partial^{2} T_{1}}{\partial z^{2}}+\alpha\left(1-R_{1}\right) I(t) \exp (-\alpha z) \frac{\left[1+R_{2} \exp \left(2 \alpha\left(z-h_{1}\right)\right)\right]}{\left[1-R_{1} R_{2} \exp \left(-2 \alpha h_{1}\right)\right]}, \\
\rho_{2}\left[c_{2}+H_{f} \delta\left(T_{2}-T_{f}\right)\right] \frac{\partial T_{2}}{\partial t}=\lambda_{2} \frac{\partial^{2} T_{2}}{\partial z^{2}}+\rho_{2} Q Z \exp \left(-\frac{E}{R T_{2}}\right) .
\end{gathered}
$$

with the following initial and boundary conditions

$$
\begin{gathered}
T_{1}(z, 0)=T_{2}(z, 0)=T_{0}, \frac{\partial T_{1}(0, t)}{\partial z}=\frac{\partial T_{2}\left(h_{1}+h_{2}, t\right)}{\partial z}=0, \\
\lambda_{1} \frac{\partial T_{1}\left(h_{1}, t\right)}{\partial z}=\lambda_{2} \frac{\partial T_{2}\left(h_{1}, t\right)}{\partial z}, T_{1}\left(h_{1}, t\right)=T_{2}\left(h_{1}, t\right) .
\end{gathered}
$$

Here $h_{1}, T_{1}$ is the thickness and the temperature of the oxide film; $h_{2}, T_{2}$ is the thickness and the temperature of PETN; $T_{0}$ is the initial temperature of the two-layer systems, i.e. the copper oxide and PETN; $\lambda_{1}, c_{1}, \lambda_{2}, c_{2}$ are coefficients of thermal conductivity and thermal capacitance of the copper oxide and PETN; $\rho_{1}, \rho_{2}$ is the density of the copper oxide and PETN; $R_{1}, R_{2}$ are reflection coefficients of the light flux off outer and inner surfaces of the copper oxide; $I$ is the light intensity; $H_{f}, T_{f}$ is the latent heat of melting and the melting temperature of PETN; $\delta\left(T_{2}-T_{f}\right)$ is the delta function; $Q, Z, E$ is the heat of reaction per unit of mass, the frequency factor and the activation energy of the rate of thermal decomposition; $R$ is the universal gas constant.

Equation (1), for the film absorbing the laser radiation, is written taking into account the multiple reflection of the light flux from the opposite sides of the film. The thermal conductivity equation (2) for explosives is written considering its melting point. The melting point of PETN substantially lower than the ignition temperature. External heat sink wasn't taken into account in this problem. It was assumed that the laser pulse duration and the delay time of ignition is much smaller than the characteristic time of the external heat sink. An additional assumption was made that thermophysical properties of PETN and the melt are similar.

Dependence of the intensity of the light flux from time was given in a form of a rectangular pulse:

$$
\begin{gathered}
I(t)=W_{i} / \tau_{i}=I_{0}, \tau_{i} \geq t \geq 0 ; \\
I(t)=0, \tau_{i}>0,
\end{gathered}
$$

where $W_{i}=72.2 \cdot 10^{4} \mathrm{~J} / \mathrm{m}^{2}$ is the energy density of the laser pulse; $\tau_{i}=20 \cdot 10^{-3} \mathrm{~s}$ is the laser pulse duration. Absorption of the light flux that passed through an absorbing film was neglected in the PETN due to the small absorption coefficient of explosive in this region of the spectrum.

\section{Results of numerical calculations and discussion}

In the numerical solution of equations (1) and (2) with the boundary conditions (3) implicit difference schemes were used, which were solved by the sweep method [16]. Size of steps in time and space were chosen so that the error in the law of conservation of energy was smaller then $\sim 7 \%$. Melting algorithm for solving the thermal conductivity equation for explosive is described in $[10,11,13]$. Arrhenius nonlinearity was linearized at each time step using the Frank - Kamenetz transformation:

$$
\exp \left(-E / R T_{i}\right) \approx \exp \left(-E / R \breve{T}_{i}\right) \exp \left(-E \Delta T_{i} / R \breve{T}_{i}^{2}\right) \approx\left(1+\frac{E T_{i}}{R \breve{T}_{i}^{2}}-\frac{E}{R \breve{T}_{i}}\right) \exp \left(-E / R \breve{T}_{i}\right),
$$


where $T_{i}, \breve{T}_{i}$ is the temperature of calculated and the previous time layer in the $i$-th cell of the difference scheme in the coordinate $z$.

Actual thickness of explosive is much greater than the thickness of the film. Therefore, to minimize the calculation time the number of divisions of $z$ coordinate was chosen that on the right boundary film-explosive the temperature at the time of ignition of PETN was equal $T_{0}$. Step along $z$ coordinate was set independently from the thickness of the film and was always the same.

Solution of the thermal conductivity equation for copper oxide were performed under the following thermophysical parameters and density: $\lambda_{1}=1.013 \mathrm{~W} /(\mathrm{m} \cdot \mathrm{K}), c_{1}=6 \cdot 10^{2} \mathrm{~J} /(\mathrm{kg} \cdot \mathrm{K})$, $\rho_{1}=6.45 \cdot 10^{3} \mathrm{~kg} / \mathrm{m}^{3}$ [17]. The absorption coefficient of the film was assumed to be $\alpha=4200 \cdot 10^{2}$ $\mathrm{m}^{-1}$. Reflection coefficients from opposite sides of the film were assumed to be $R_{1}=R_{2}=0.5$. Calculations for PETN were performed under the following thermophysical and kinetic parameters: $\lambda_{2}=2.51 \cdot 10^{-1} \mathrm{~W} /(\mathrm{m} \cdot \mathrm{K}), \rho_{2}=1.77 \cdot 10^{3} \mathrm{~kg} / \mathrm{m}^{3}, E=196.6 \mathrm{~kJ} / \mathrm{mol}, Z=6.3 \cdot 10^{19} \mathrm{~s}^{-1}, Q=1.26$ $\mathrm{MJ} / \mathrm{kg}, c_{2}=1.26 \cdot 10^{3} \mathrm{~J} /(\mathrm{kg} \cdot K), H_{f}=192 \mathrm{~kJ} / \mathrm{kg}, T_{f}=413 \mathrm{~K}[9-11,18]$.

Some of the results of numerical calculations are shown on Figure 2 - Figure 4. Calculations shown that dependence of threshold energy of ignition $W^{*}$ of PETN by a laser pulse has a linear dependence from the thicknesses of the absorbing film (Figure 2). This is caused by the fact that the thicker the film that absorbs light the more energy is needed for its heating in order to reach critical temperature at the film-explosive boundary. Calculations shown that critical the temperature $\Delta T^{*}$ on the boundary of two materials doesn't depend from the thickness of copper oxide and by the end of a laser pulse with threshold density is approximately equal to $270 \mathrm{~K}$. Ignition of the explosive occurs at the end of the laser pulse.

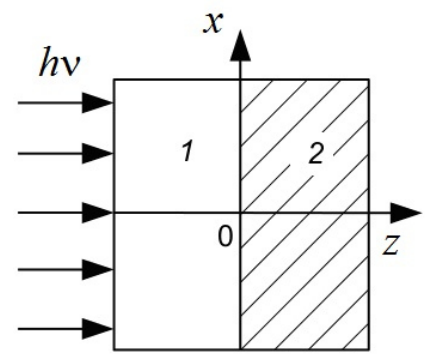

Figure 1. The two-layer system: 1 - copper oxide film 2 - PETN.

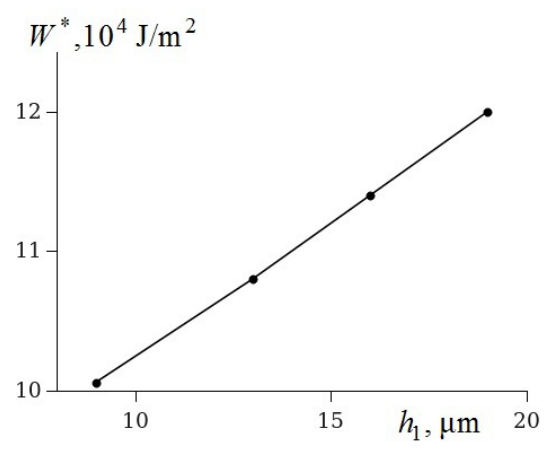

Figure 2. The dependence of the threshold energy ignition PETN laser pulse, depending on the thickness of the absorbing film.

Figure 3 and Figure 4 show results of calculations of the dynamic of the temperature distribution in the system film-PETN at thicknesses heated by a light pulse from 9 microns and 19 microns films 
respectively. Calculations results show that at the end of the laser pulse the temperature in a film decreases due to the heat transfer to the explosive what leads to the ingition of PETN after a certain time. Maximum temperature of the explosive at the moment of ignition is found not at the boundary film-explosive but at some distance from the boundary.

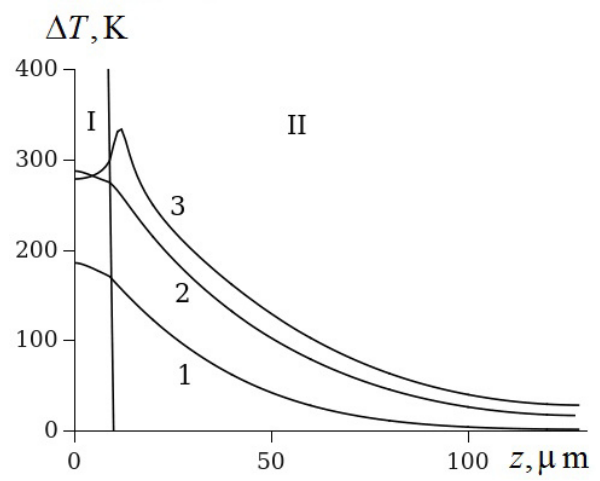

Figure 3. The dynamics of the distribution of temperature in the film (I) and PETN (II) with a film thickness $h_{1}=9 \mu \mathrm{m}$, and time $t=10$ (1), 20 (2) and $24.16 \mathrm{~ms}$ (3).

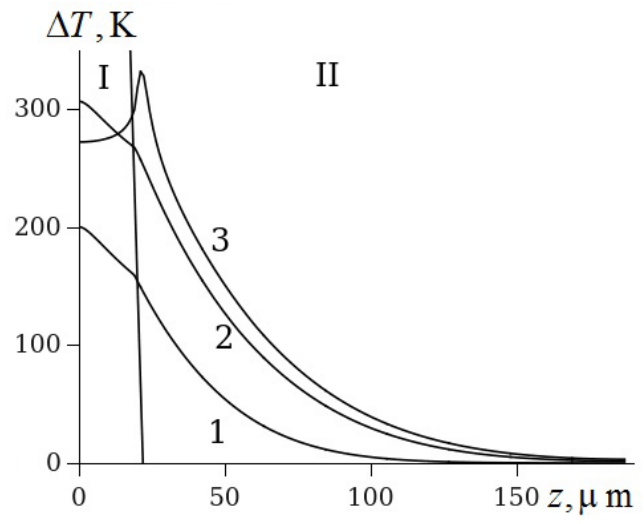

Figure 4. The dynamics of the distribution of temperature in the film (I) and PETN (II) with a film thickness $h_{1}=19 \mu \mathrm{m}$, and time $t=10$ (1), 20 (2) and $22.97 \mathrm{~ms} \mathrm{(3).}$

Figure 3 and Figure 4 show that the ignition delay time of PETN by the 19 microns thick film less than the ignition delay time of PETN by the 9 microns film. The reason is that the thicker contain more heat then in the thinner one. These calculations are consistent with [19], which presents results for numerical simulation of a solid-phase ignition of metallized condensed material heated to high temperatures by a particle.

\section{Conclusions}

Thus, calculations shown: 1 - the increase of the thickness of the film that absorbs laser radiation leads to an increase of the threshold energy of the ignition of PETN; 2 - critical temperature of the ignition of PETN on the boundary of film-explosive almost doesn't depend on the thickness of the film; 3 - the thicker film has shorter ignition delay time of PETN near the threshold at the end of a laser pulse in comparison to the thinner film.

The work has been carried out within the framework of the government assignment in science No. $3.1235 .2014 \mathrm{~K}$. 


\section{References}

1. K. Nagayama, Y. Kotsuka, M. Nakahara, S. Kubota, Science and Technology of Energetic Materials 66, 416 (2005)

2. A.V. Khaneft, E.V. Duginov, Energetic Materials - Characterisation and Performance of Advanced Systems. 38 th International Annual Conference of ICT june 26 - june 29, Karlsruhe, Federal Republic of Germany. 117 (2007)

3. E.D. Aluker, N.L. Aluker, G.M. Belokurov, A.G Krechetov, B.G. Loboiko, D.R. Nurmukhametov, A.V. Tupitsyin, V.P. Filin, Russian Journal of Physical Chemistry B 4, 63 (2010)

4. E.D. Aluker, A.S. Zverev, A.G. Krechetov, A.Yu. Mitrofanov, A.O. Terent'eva, A.V. Tupitsyn, Yu.P. Sakharchuk, Russian Physics Journal 56,1357 (2014)

5. R.S. Burkina, V.V. Medvedev, O.V. Khrenova, Combust. Expl. Shock Waves, 46, 554 (2010)

6. R.S. Burkina, E.Yu. Morozova, V.P. Tsipilev, Combust. Expl. Shock Waves 47, 581 (2011)

7. V.E. Zarco, V.N. Simonenko, P.I. Kalmykov, A.A. Kvasov, E.N. Chesnokov, K.E. Kuper, Combust. Expl. Shock Waves 45, 752 (2009)

8. I.G. Assovskii, V.V. Kozynda, Docl. Akad. Nauk 442, 771 (2012)

9. E.V. Duginov, A.V. Khaneft, Combust. Expl. Shock Waves 47, 490, (2011)

10. A.V. Khaneft, E.V. Duginov, Combust. Expl. Shock Waves 48, 699 (2012)

11. A.V. Khaneft, V.A. Dolgachev, Combust. Expl. Shock Waves 50, 105 (2014)

12. M.A. Ilyushin, I.A. Ugryumov, A.S. Kozlov, V.Yu. Dolmatov, I.V. Shugalei, A.N. Golovchak, A.V. Vedenetskii, D.V. Korolev, V.B. Ostashev, Khim. Fiz. 24, 49 (2005)

13. V.A. Dolgachev, A.V. Khaneft, Chemical Physics and mezoskopiya 14, 536 (2012) [in Russian]

14. E.D. Aluker, A.G. Krechetov, A.Yu. Mitrofanov, A.S. Zverev, M.M. Kuklja, J. Phys. Chem. C. 116, 2482 (2012)

15. D.O. Glushkov, G.V. Kuznetsov, P.A. Strizhak. Combust. Expl. and Shock Waves 50, 670 (2014)

16. A.A. Samarskii, Yu.P. Popov, Difference methods for solving problems of gas dynamics. (Nauka, Moscow, 1992) [in Russian]

17. Yu.E. Sheludyak, L.Ya. Kashporov, L.A. Malinin, V.N. Tsalkov, Thermal properties of combustible systems components (Inform TEI, Moscow, 1992) [in Russian]

18. L.P. Orlenko, Explosion Physics, (Nauka, Moscow, 2002) [in Russian]

19. O.D. Glushkov, G.V. Kuznetsov, P.A. Strizhak, Russian Journal of Physical Chemistry B 5, 1000, (2011) 